\title{
An Evaluation Method for Commutation Failure Caused by Waveform Distortion
}

\author{
Hao Zhou ${ }^{1}$, Can $\mathrm{Su}^{2}$ and Hao Zhou ${ }^{*}$ \\ ${ }^{1}$ Electric Power Research Institute of State Grid Hebei Electric Power Company, Shijiazhuang, Hebei,050000, China \\ ${ }^{2}$ Electric Power Research Institute of State Grid Hebei Electric Power Company, Shijiazhuang, Hebei,050000, China
}

\begin{abstract}
Commutation failure is a key issue that affects the safe operation of HVDC transmission systems. Scholars at home and abroad have conducted research on the mechanism, evaluation indicators, and control strategies of commutation failure. However, most of the researches are aimed at the working conditions of commutation failure caused by a short-circuit fault on the AC side. There are few studies on the commutation failure caused by the harmonic injection on the AC side and the distortion of the AC bus voltage waveform. This paper clarifies the mechanism of commutation failure caused by waveform distortion, and proposes an index of harmonic commutation margin. On this basis, an evaluation method for commutation failure caused by waveform distortion is proposed. By predicting the commutation voltage-time area and optimization methods to assess whether the current voltage conditions have the risk of commutation failure. Finally, an electromagnetic transient simulation model is established on PSCAD/EMTDC, and the evaluation method in this chapter is verified by simulating the working conditions of the inverter side bus voltage waveform distortion. The results show its effectiveness in evaluating the commutation failure. The research provides a theoretical basis to the implementation of the control function and has broad engineering application prospects.
\end{abstract}

\section{Introduction}

Compared with the AC transmission, HVDC has better economic benefits in long distance, high-capacity transportation, wide application in power system networking ${ }^{[1]}$.HVDC projects have played a vital role in China. Commutation failure is one of the common faults of HVDC systems, which causes DC systems to be disturbed, and the DC current rapidly increases. Moreover, the reactive power consumption of the converter is increased, affecting the life of the converter valve. Therefore, it is of great significance to effectively master the characteristics of the commutation failure, which effectively solves the problem of commutation failure in actual projects.

Scholars at home and abroad have studied the mechanism, assessment indicators, and suppression measures of commutation failure. One study [2] defines the necessary time-voltage area for the commutation process, and when the actual time-voltage area is less than the threshold, it is determined that commutation failed, but the measurement of the phase start time causes its results to be conservative.

Another study [3] proposed an advanced firing angle inner loop controller for the asymmetry fault on the inverter side to effectively suppress continuous commutation failures. For symmetrical and asymmetrical faults, Commutation Prevention Control (CFPREV) is proposed ${ }^{[4]}$. Its drawback is that continuously increasing the firing angle will limit the transmission power and it ignores the influence of the voltage waveform distortion caused by harmonic. While the CFPREV detection method has the defect that the zero-sequence voltage detection starts slowly when the AC voltage crosses zero. Based on [4], The sin-cos component detection discrimination is added to the original method ${ }^{[5]}$. One study[6] proposes an improved CFPREV control strategy that integrates current and voltage criteria, which improves the speed and sensitivity of fault detection. But most studies on commutation failure are designed for $\mathrm{AC}$ system short-circuit fault conditions and most of the criteria of these methods are based on the amplitude of the voltage, so it is impossible to consider the influence of voltage waveform distortion such as harmonics on the commutation process in the transient process of the system $^{[7]}$. One study [8] simulates different degrees of two-phase and three-phase $\mathrm{AC}$ bus voltage waveform distortion by switching a set of AC filters on the Tianguang DC RTDS platform. The results show that for the weak AC system on the inverter side, the voltage distortion fault caused by the voltage non-zero-crossing point closing is easy to cause the commutation failure, in severe cases the CFPREV control cannot avoid the commutation failure. One study [9] uses an optimized method to present the index LTHD, which characterizes the influence of the magnetizing inrush current on commutation, and provides a quantitative basis for solving the commutation failure caused by waveform distortion. However, there exists an error in the harmonic estimation

"Corresponding author's e-mail: dyy_suc@he.sgcc.com.cn 
analysis method based on Fourier analysis, and the performance of the algorithm is not good. Therefore, it is of great significance to study the influence mechanism of voltage waveform distortion on commutation and propose corresponding evaluation indicators to implement the control effect in advance.

In this paper, the harmonic commutation margin is proposed to characterize the maximum disadvantage of waveform distortion to the commutation area under the premise of successful commutation. The commutation area is predicted by the method of numerical integration. Based on this, the commutation area is solved by an optimized method. The maximum line-line voltage change allowed under the premise of phase success is used as the threshold value, which is compared with the actual sampled value as an evaluation method for whether commutation failure will occur. Moreover, the paper establishes an electromagnetic transient simulation model in PSCAD/EMTDC, and compares with the traditional commutation failure predictive control simulation, it is verified that the evaluation method in this paper can accurately assess whether the current commutation voltage situation has the risk of commutation failure. The research provides a theoretical basis to the implementation of the control function and has broad engineering application prospects.

\section{Materials and Methods}

\subsection{Mechanism of LCC-HVDC Commutation Failure Caused by Waveform Distortion}

During the commutation process, if the valve that exits the conduction fails to close in time under the action of the reverse voltage, or when the voltage is reversed, the commutation phenomenon occurs to the valve that is scheduled to be turned off, the commutation fails.

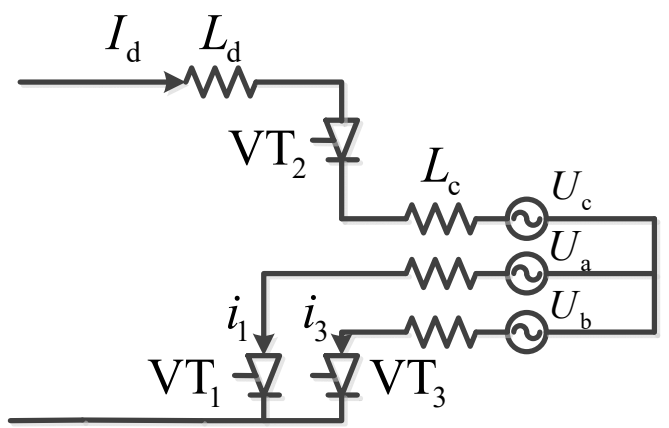

Figure 1. Six-pulse converter bridge equivalent circuit diagram

Figure 1 shows the equivalent circuit of the commutation process of a standard six-pulse converter bridge. The commutation from VT1 to VT3 is being completed, according to Kirchhoff's law:

$$
\begin{gathered}
L_{\mathrm{c}} \frac{\mathrm{d} i_{3}}{\mathrm{~d} t}+U_{\mathrm{c}}=L_{\mathrm{c}} \frac{\mathrm{d} i_{1}}{\mathrm{~d} t}+U_{\mathrm{a}} \\
i_{1}+i_{3}=I_{\mathrm{d}}
\end{gathered}
$$

$i_{1}, i_{3}$ are the currents flowing through VT1 and VT3 respectively, and $L_{\mathrm{c}}$ is the commutation reactance converted to the valve side; $I_{\mathrm{d}}$ is the direct current to be transmitted. From equations (1) and (2),

$$
U_{\mathrm{ca}}=2 L_{\mathrm{c}} \frac{\mathrm{d} i_{1}}{\mathrm{~d} t}
$$

Integrate both ends of equation (3). The lower limit of the integral is the time when the commutation starts $\alpha / \omega$,and the upper limit of the integral is the time when the commutation ends $(\alpha+\mu) / \omega$ 。 For thyristor VT1, the current flowing through it at the beginning of commutation is 0 , and the current flowing through it at the end of commutation is $I_{\mathrm{d}}$,

$$
I_{\mathrm{d}}=\int_{\alpha / \omega}^{(\alpha+\mu) / \omega} \frac{U_{\mathrm{ca}}}{2 L_{\mathrm{c}}} \mathrm{d} t
$$

In actual systems, constant current control is often used on the rectifier side, that is, the transmitted DC current is constant. This formula shows that the commutation voltage required for the commutation process is constant with respect to the area of time, which is determined by the DC current $I_{\mathrm{d}}{ }^{[10]}$.

Short-circuit faults in the system, such as no-load closing of transformers during transmission and distribution, some non-linear electrical equipment will generate harmonics in the power system, which will be amplified during the propagation process, resulting in the occurrence of voltage waveforms on the commutation bus. Distortion is no longer a standard sine wave.

$$
U=U_{0}+\sum_{n=1}^{N} U_{\mathrm{n}} \sin \left(n \omega t+\varphi_{\mathrm{n}}\right)
$$

$U_{0}$ is the DC component of the voltage, $U_{\mathrm{n}}, \varphi_{\mathrm{n}}$ are the fundamental wave and the amplitude and phase of each harmonic voltage. At this time, the formula (4) can be expressed as:

$$
I_{\mathrm{d}}=\int_{t_{0}}^{t_{1}} \frac{U_{0}}{2 L_{\mathrm{c}}} d t+\sum_{n=1}^{N} \int_{t_{0}}^{t_{1}} \frac{U_{\mathrm{n}} \sin \left(n \omega t+\varphi_{\mathrm{n}}\right)}{2 L_{\mathrm{c}}} d t
$$

Among them, $t_{0}=\alpha / \omega, t_{1}=(\pi-\delta-\gamma) / \omega$ respectively represent the start and end moments of the commutation process, and $\delta$ is the offset angle of the line voltage zerocrossing point caused by voltage distortion, which is represented by $U_{0}, U_{\mathrm{n}}, \varphi_{\mathrm{n}}$ uniquely. If the commutation is successful, $\omega t_{1}=\alpha+\mu \leq \pi-\gamma_{\min }$, if the equation does not hold, that is, the integral of the fundamental wave voltage and each harmonic voltage on the right side of equation (6) is not enough to make the thyristor 4 When the current drops from $I_{\mathrm{d}}$ to 0 , the thyristor 4 will continue to conduct at this time, and commutation failure occurs.

\subsection{An evaluation method of commutation failure caused by waveform distortion}

In order to propose an evaluation method for commutation failure caused by waveform distortion, it is necessary to find the conditions for successful commutation. The value of the extinction angle is a determining factor for measuring the success of the commutation failure, but in the case of voltage waveform distortion, it is difficult to accurately predict the value of the extinction angle. Starting from the theory of commutation time-voltage area, it can be obtained from equation (6) that during the 
commutation process, the required commutation timevoltage area is constant. The voltage amplitude and phase angle of each harmonic satisfies:

$$
2 L_{\mathrm{c}} I_{\mathrm{d}}=\int_{t_{0}}^{t_{1}} U_{0} d t+\sum_{n=1}^{N} \int_{t_{0}}^{t_{1}} U_{\mathrm{n}} \sin \left(n \omega t+\varphi_{\mathrm{n}}\right) d t
$$

Among them, $t_{0}=\alpha / \omega, t_{1}=(\pi-\delta \quad-\gamma) / \omega$ respectively represent the starting and ending moments of the commutation process. If the commutation is to be successful, $t_{1}$ must satisfy: $\omega t_{1}=\alpha+\mu \leq \pi-\gamma_{\min }$, that is, the commutation needs to be completed before $\left(\pi-\gamma_{\min }\right) / \omega$. Under the premise of ensuring the success of commutation, the maximum value of the effects of the factors that are not conducive to the commutation, such as the fundamental voltage drop, the waveform distortion caused by the harmonic voltage, etc., is defined as the harmonic commutation margin $\mathrm{M}$ :

$$
M=2 L_{\mathrm{c}} I_{\mathrm{d}}-\int_{\frac{\alpha}{\omega}}^{\frac{\pi-\gamma_{\min }}{\omega}} U_{\mathrm{IN}} \sin \left(\omega t+\varphi_{\mathrm{n}}\right) d t=\int_{\frac{\alpha}{\omega}}^{\frac{\pi-\gamma_{\min }}{\omega}} U_{0} d t+\sum_{n=1}^{N} \int_{\frac{\alpha}{\omega}}^{\frac{\pi-\gamma_{\min }}{\omega}} U_{\mathrm{n}} \sin \left(n \omega t+\varphi_{\mathrm{n}}\right) d t
$$

Among them, $U_{1 \mathrm{~N}}$ is the fundamental wave amplitude of the line-line voltage. It can be seen from (8) that the physical meaning of $\mathrm{M}$ is the maximum value of the area of voltage versus time in the commutation process that reduces the factors that are not conducive to commutation. When the actual harmonic time-voltage area is less than $\mathrm{M}$, it means that the prolonged commutation time will not make the actual extinction angle less than the minimum extinction angle, that is, no commutation failure will occur, otherwise the risk of commutation failure is considered. When the firing angle $\alpha$ changes, the value of $\mathrm{M}$ changes accordingly.

In the case of waveform distortion (not sine wave), due to the unpredictable influence of the commutation voltage, it is necessary to effectively predict the harmonic commutation time-voltage area, and define the commutation bus commutation voltage change $\Delta u(t)$ caused by the waveform distortion at $\mathrm{t}$ is:

$$
\Delta u(t)=u_{\text {nor }}-u_{\text {act }}
$$

Among them, $u_{\text {nor }}$ is the instantaneous voltage value at $\mathrm{t}$ under undistorted conditions, and $u_{\text {act }}$ is the actual voltage sampled value. At t, $\Delta u(t)$ can be obtained by sampling the commutation bus voltage and expressed as the sum of harmonics:

$$
\Delta u(t)=U_{0}+\sum_{i=1}^{n} U_{\mathrm{i}} \sin \left(i \omega t+\varphi_{\mathrm{i}}\right)
$$

For any time $t+\Delta t$ in the future, the line-line voltage variation $\mathrm{u}(t+\Delta t)$ satisfies:

$$
u(t+\Delta t)=U_{0}+\sum_{i=1}^{n} U_{\mathrm{i}} \sin \left[i \omega(t+\Delta t)+\varphi_{\mathrm{i}}\right]
$$

Let $K_{i}=\sin \left(i \omega t+\varphi_{i}\right)$, use the trigonometric formula to simplify equation (11):

$$
u(t+\Delta t)=U_{0}+\sum_{i=1}^{n} U_{\mathrm{i}}\left[K_{\mathrm{i}} \cos (i \omega \Delta t)+\sqrt{1-K_{\mathrm{i}}^{2}} \sin (i \omega \Delta t)\right]
$$

Among them, $\cos (i \omega \Delta t), \sin (i \omega \Delta t)$ are known quantities, so the line-line voltage change at any time in the future can be expressed by $\mathrm{Ui}, \mathrm{Ki}$, and then the harmonic conversion can be expressed by the method of numerical integration. Take the trapezoidal integration method as an example. As shown in Figure $2, t_{0}$ is the time corresponding to the natural commutation point, $t$ is the current sampling time, and $t_{1}$ and $t_{2}$ are the start and end times of the commutation respectively. Suppose the time intervals between the start and end times of the commutation and the sampling point are $\Delta t_{1}$ and $\Delta t_{2}$, respectively, and $u\left(t+\Delta t_{1}\right)$ and $u\left(t+\Delta t_{2}\right)$ can be obtained by (12), then the commutation time of the harmonics-the voltage area $\mathrm{S}$ is:

$$
S=\frac{\left(\Delta t_{2}-\Delta t_{1}\right) \cdot\left[u\left(t+\Delta t_{1}\right)+u\left(t+\Delta t_{2}\right)\right]}{2}
$$

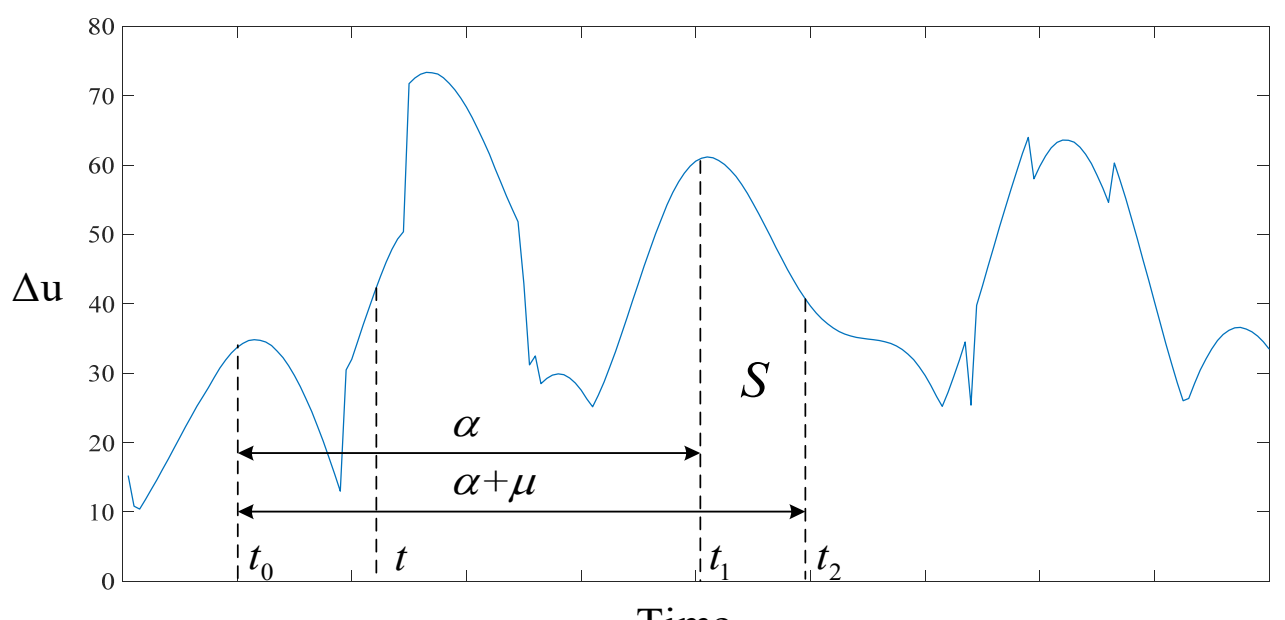

Time

Figure 2. Schematic diagram showing the area of harmonic commutation by numerical integration

At $t$, the line-line voltage change $\Delta u(t)$ can be calculated in real time by sampling the voltage value of the commutation bus. Based on the above derivation, the optimization method is used to find out that the next commutation is successful, allowing Waveform distortion causes the maximum absolute value of the line-line voltage change at $\mathrm{t}:\left|\Delta u_{\max }(t)\right|$, when $\left|\Delta u_{\max }(t)\right|<|\Delta u(t)|$ indicates that there is a risk of commutation failure. In 
summary, the optimized model for evaluating commutation failure caused by waveform distortion is as follows:

$$
\begin{aligned}
& \text { obj. } \max |\Delta u(t)|=\left|\sum_{i=1}^{n} U_{\mathrm{i}} \sin \left(i \omega t+\varphi_{\mathrm{i}}\right)\right|=\mid \sum_{i=1}^{n} U_{\mathrm{i}} K_{\mathrm{i}} \\
& \text { s.t. } \\
& \qquad \begin{array}{c}
\mathrm{S}<\mathrm{M} \\
U_{1 \min } \leq U_{1} \leq U_{1 \mathrm{~N}}, 0 \leq U_{\mathrm{n}} \leq U_{\mathrm{nmax}} \\
-1 \leq K_{i} \leq 1
\end{array}
\end{aligned}
$$

(1) Harmonic commutation voltage-time area constraint: In order to ensure successful commutation, the harmonic commutation time-voltage area should be smaller than the harmonic commutation margin.

(2) Voltage amplitude constraint: For the $n$th harmonic voltage, its harmonic commutation area can be expressed as:

$$
\int_{\frac{\alpha}{\omega}}^{\frac{\pi-\gamma}{\omega}} U_{\mathrm{n}} \sin \left(n \omega t+\varphi_{\mathrm{n}}\right) d t=\frac{U_{\mathrm{n}}}{n \omega}\left[2 \sin \left(\theta_{\mathrm{n}}+\varphi_{\mathrm{n}}\right) \sin \left(\frac{n \pi-n \alpha-n \gamma}{2}\right)\right]
$$

Among them, $\theta_{n}$ is an additional angle to simplify the process. It can be seen from equation (15) that the maximum possible harmonic commutation area of the nth harmonic is

$$
S_{\text {nmax }}=\frac{2 U_{\mathrm{n}}}{n \omega} \sin \left(\frac{n \pi-n \alpha-n \gamma_{\min }}{2}\right)
$$

In order to ensure the success of commutation, it is necessary to satisfy that each maximum harmonic voltagetime area is less than the commutation margin $\mathrm{M}$ :

$$
\begin{aligned}
& U_{n} \leq n \omega \mathrm{M} / 2 \sin \left(\frac{n\left(\pi-\alpha-\gamma_{\min }\right)}{2}\right) \\
& U_{n \max }=n \omega \mathrm{M} / 2 \sin \left(\frac{n\left(\pi-\alpha-\gamma_{\min }\right)}{2}\right)
\end{aligned}
$$

For the fundamental wave voltage, the drop of the fundamental wave voltage is not conducive to commutation. The lower limit of the fundamental wave voltage should meet the conditions for successful commutation. Therefore,

$$
\int_{\alpha / \omega}^{\left(\pi-\gamma_{\min }\right) / \omega} \frac{U_{1 \min } \sin (\omega t)}{2 L_{\mathrm{c}}} \mathrm{d} t=I_{\mathrm{d}}
$$

$$
U_{1 \text { min }}=\frac{2 I_{\mathrm{d}} \omega L_{\mathrm{c}}}{\cos \alpha-\cos \left(\pi-\gamma_{\min }\right)}
$$

(3) Phase constraint: Since the initial phase of each harmonic satisfies: $-\pi \leq \varphi_{i} \leq \pi$, so $-1 \leq K_{i} \leq 1$.

Study [10] proposes that the harmonic commutation coefficient $A_{\mathrm{n}}$ is used to evaluate the maximum possible influence of the nth harmonic on the commutation process in a certain operating state, because the harmonic impedance $(n \omega L)$ is proportional to the harmonic order, as the harmonic order increases, $A_{\mathrm{n}}$ shows a decreasing trend. Therefore, low-order harmonics play a major role in the commutation failure of DC systems. Therefore, the accuracy of the results can be satisfied by considering the low-frequency harmonics in the main frequencies in the model.

\section{Results \& Discussion}

The simulation model of this paper is established based on the CIGRE HVDC standard test model. The main parameters of the model are shown in Table 1. By connecting a harmonic voltage source in series with the equivalent power supply on the $\mathrm{AC}$ side to simulate the working conditions of the voltage waveform distortion of the commutation bus, the amplitude and phase of each harmonic are shown in Table 2:

Table 1. Main parameters of the simulation system.

\begin{tabular}{cc}
\hline System parameters & Value \\
\hline L-G voltage $(\mathrm{RMS}, \mathrm{kV})$ & 217 \\
Commutation reactance $(\Omega)$ & 10.85 \\
Firing angle $\left(^{\circ}\right)$ & 140 \\
Normal extinction angle $\left(^{\circ}\right)$ & 15 \\
Minimum extinction angle $\left(^{\circ}\right)$ & 7 \\
Normal direct current $(\mathrm{kA})^{\circ}$ & 2 \\
\hline
\end{tabular}

Table 2. $N$ th harmonic parameters (A- Amplitude, P-Phase)

\begin{tabular}{ccccccc}
\hline \multirow{2}{*}{ Nth harmonic } & \multicolumn{2}{c}{ Phase A } & \multicolumn{2}{c}{ Phase B } & \multicolumn{2}{c}{ Phase C } \\
\cline { 2 - 7 } & $\mathrm{A} / \mathrm{kV}$ & $\mathrm{P} /{ }^{\circ}$ & $\mathrm{A} / \mathrm{kV}$ & $\mathrm{P} /{ }^{\circ}$ & $\mathrm{A} / \mathrm{kV}$ & $\mathrm{P} /{ }^{\circ}$ \\
\hline 1 & 22.5 & 41 & 22.5 & 161 & 22.5 & -79 \\
2 & 16.6 & -20 & 16.6 & -140 & 16.6 & 100 \\
3 & 20.9 & 68 & 20.9 & -52 & 20.9 & -172 \\
4 & 19.2 & -142 & 19.2 & -22 & 19.2 & 98 \\
5 & 6.0 & 17 & 6.0 & 137 & 6.0 & -103 \\
\hline
\end{tabular}

In order to simulate the transient component on the commutation bus voltage, the attenuated DC component is superimposed on the three phases:

$$
\begin{aligned}
& U_{\mathrm{a} 0}=-10 e^{-(t-2.6)} \\
& U_{\mathrm{b} 0}=-8 e^{-(t-2.6)} \\
& U_{\mathrm{c} 0}=18 e^{-(t-2.6)}
\end{aligned}
$$

The harmonic injection time on the inverter side is $2.6 \mathrm{~s}$ and the duration is $0.08 \mathrm{~s}$. When the harmonics are injected, the three-phase voltage waveform of the inverter bus is distorted, as shown in Figure 3: 


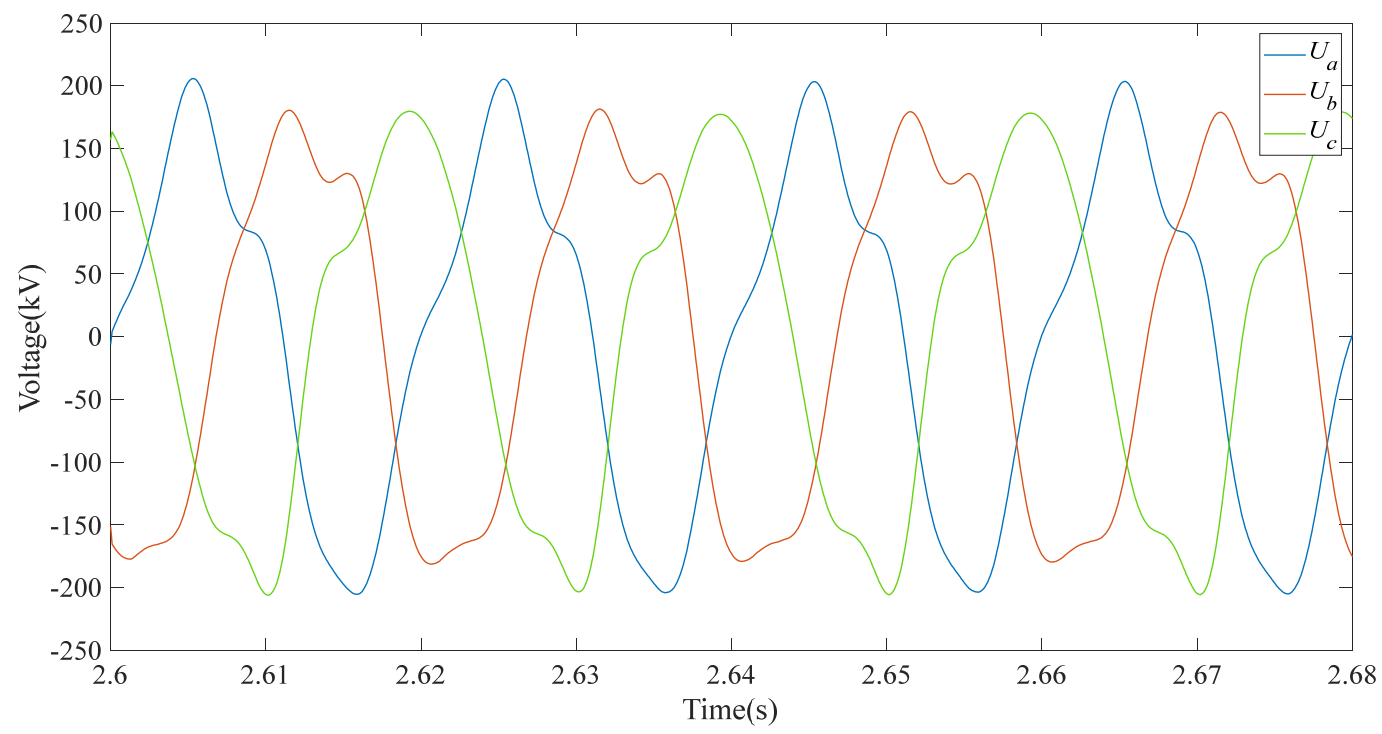

Figure 3. Diagram of three-phase voltage waveform

After the 2.6s harmonic injection, the measured values of the valve side current and the extinction angle of the converter transformer are shown in Figure 4. From the current graph on the valve side in the figure, it can be seen that a total of two commutation failures have occurred. During 2.608s-2.616s, the valve phase A current continues to be positive and the phase B current continues to be negative, as shown in Figure 1, take a six-pulse inverter as an example. In the process of commutation of thyristor VT4 to VT6, VT6 is not normally turned on, and VT1 is turned on again after being subjected to a forward voltage, that is, a commutation failure has occurred. In the same way, during $2.67 \mathrm{~s}-2.678 \mathrm{~s}$, the valve phase A current continues to be positive and the phase $\mathrm{C}$ current continues to be negative, indicating that the thyristor VT3 is in the process of commutation to VT5, VT5 is not normally turned on, and VT6 is turned on again under commutation voltage, a commutation failure occurred.

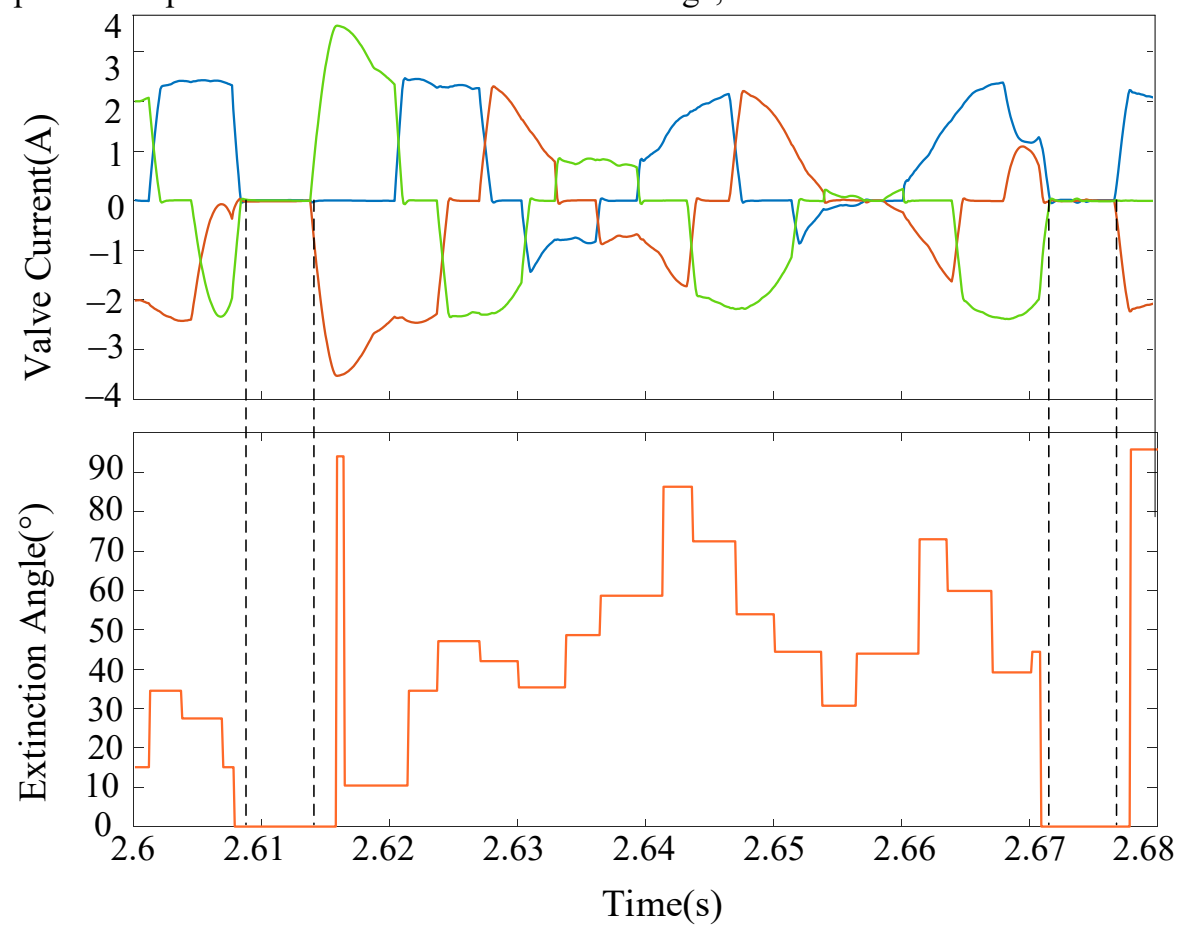

Figure 4. Waveform diagram of valve current and extinction angle

For the traditional CFPREV control, its principle and parameters refer to the setting values in the ABB software of Suzhou Station in [11]: $U_{0 \text {-ref }}=0.14, U_{\alpha \beta \text {-ref }}=0.15$.
CFPREV operation under the above conditions is simulated, and the results are shown in Figure 5. 


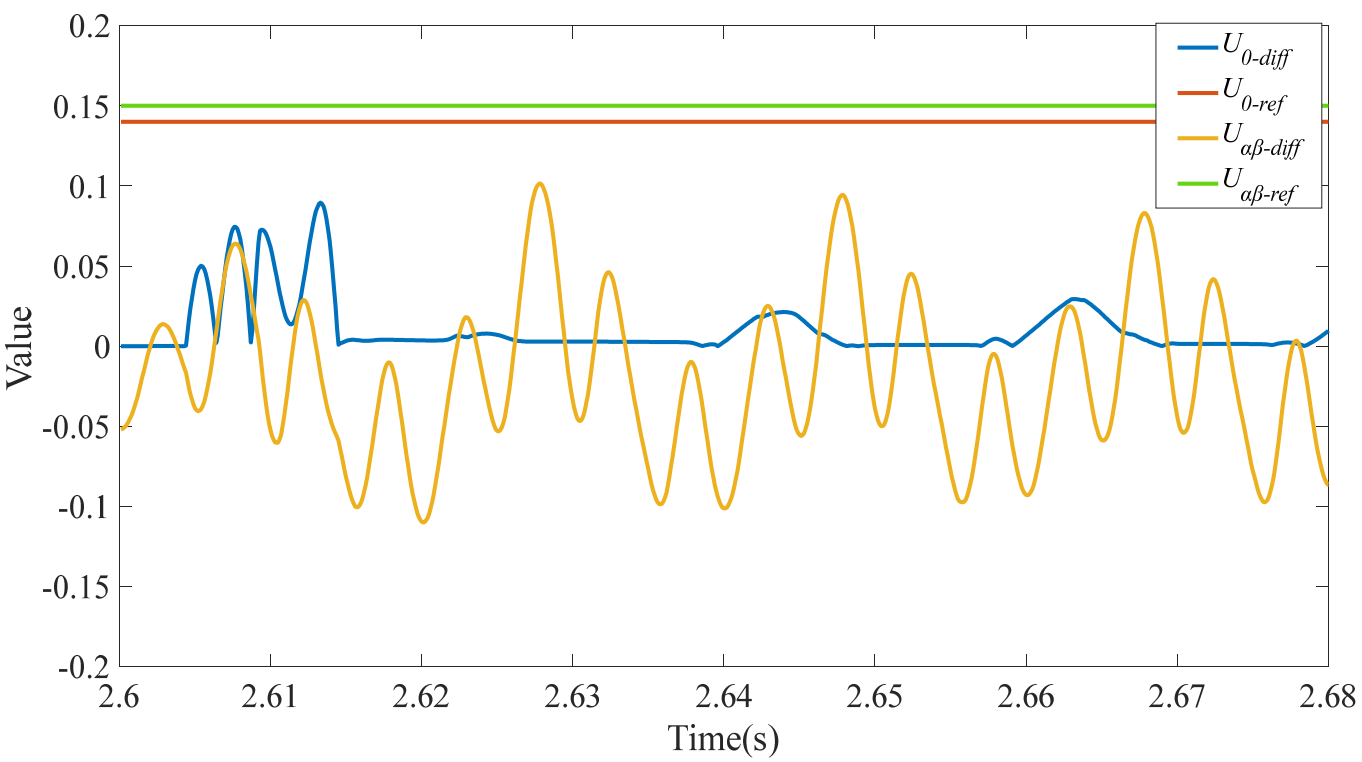

Figure 5. Schematic diagram of CFPREV parameter values

Because the three-phase harmonics are symmetrical and the voltage amplitude drops to a small degree, the above operating conditions do not meet the CFPREV single-phase criterion and three-phase criterion. Because of the threshold of the phase criterion, CFPREV will not adjust the trigger angle in time, and the original commutation failure predictive control is invalid. Using the rapid evaluation method proposed in this chapter, the optimization model takes into account the 1-5 harmonics. It can be seen from equation (18) that the upper and lower limits of the voltage are related to the size of the trigger angle, and its value is constantly changing during optimization. For example, the firing angle $\alpha=140^{\circ}$, the calculation results are shown in the following table:

Table 3. Upper limit of each harmonic amplitude.

\begin{tabular}{cc}
\hline$N$ th harmonic & $\begin{array}{c}\text { Lower limit of fundamental wave amplitude and upper limit of } \\
\text { harmonic amplitude } / \mathrm{kV}\end{array}$ \\
\hline 1 & 191.612 \\
2 & 10.558 \\
3 & 11.344 \\
4 & 12.589 \\
5 & 14.500 \\
\hline
\end{tabular}

The waveforms of the firing signal, the maximum value $U_{\max }$ of the off-line voltage change after successful commutation, and the actual line voltage change $\Delta U$ are shown in Figure 6 and Figure 7. The time when $|\Delta U|>U_{\max }$ occurs in the commutation process before $2.608 \mathrm{~s}$ and $2.67 \mathrm{~s}$, respectively Paragraph, indicating that there is a risk of commutation failure in the upcoming commutation process, and corresponding control measures need to be taken. However, the constant extinction angle control did not reduce the firing signal in time, resulting in commutation failures in $2.608 \mathrm{~s}-2.616 \mathrm{~s}$ and $2.67 \mathrm{~s}-2.678 \mathrm{~s}$. It can be seen that the algorithm proposed in this chapter is more suitable for rapid evaluation of commutation failure under voltage distortion conditions than the traditional CFPREV control. 


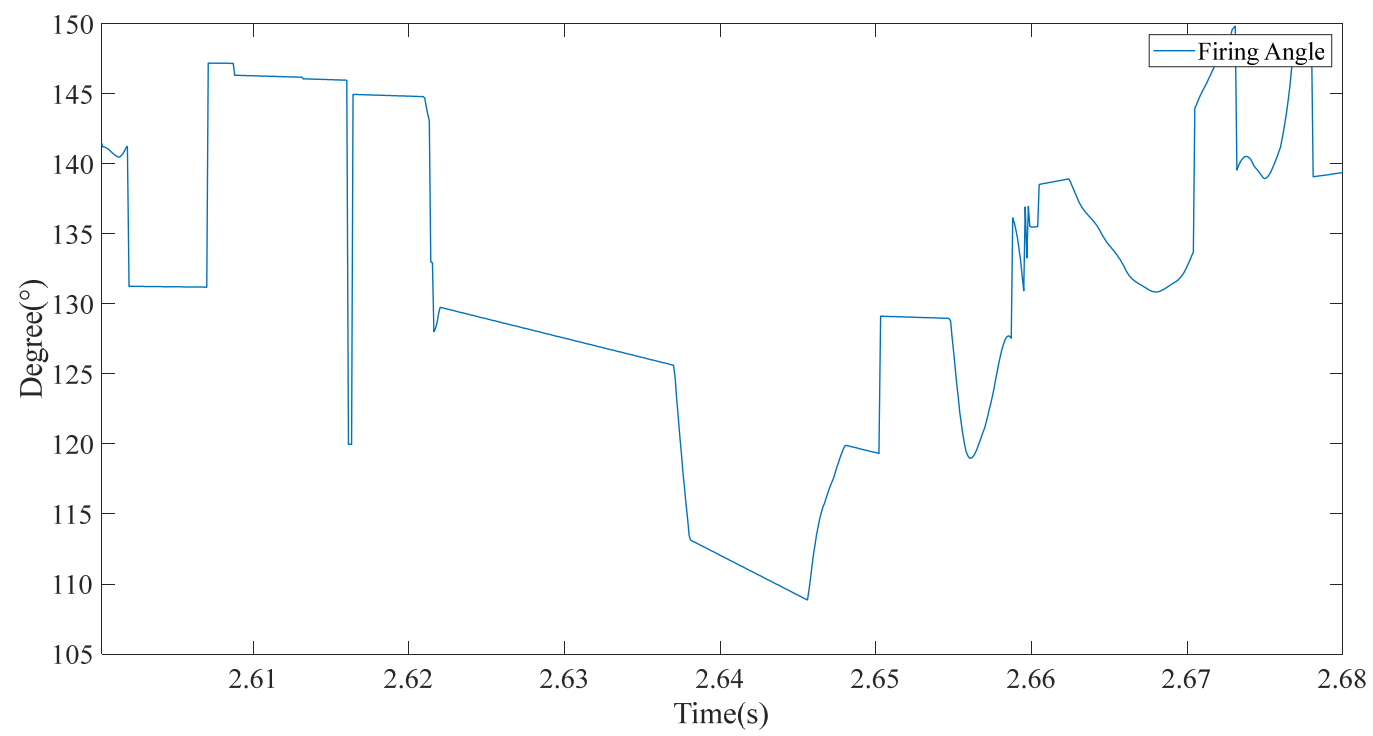

Figure 6. Firing Signal of Inverter Side

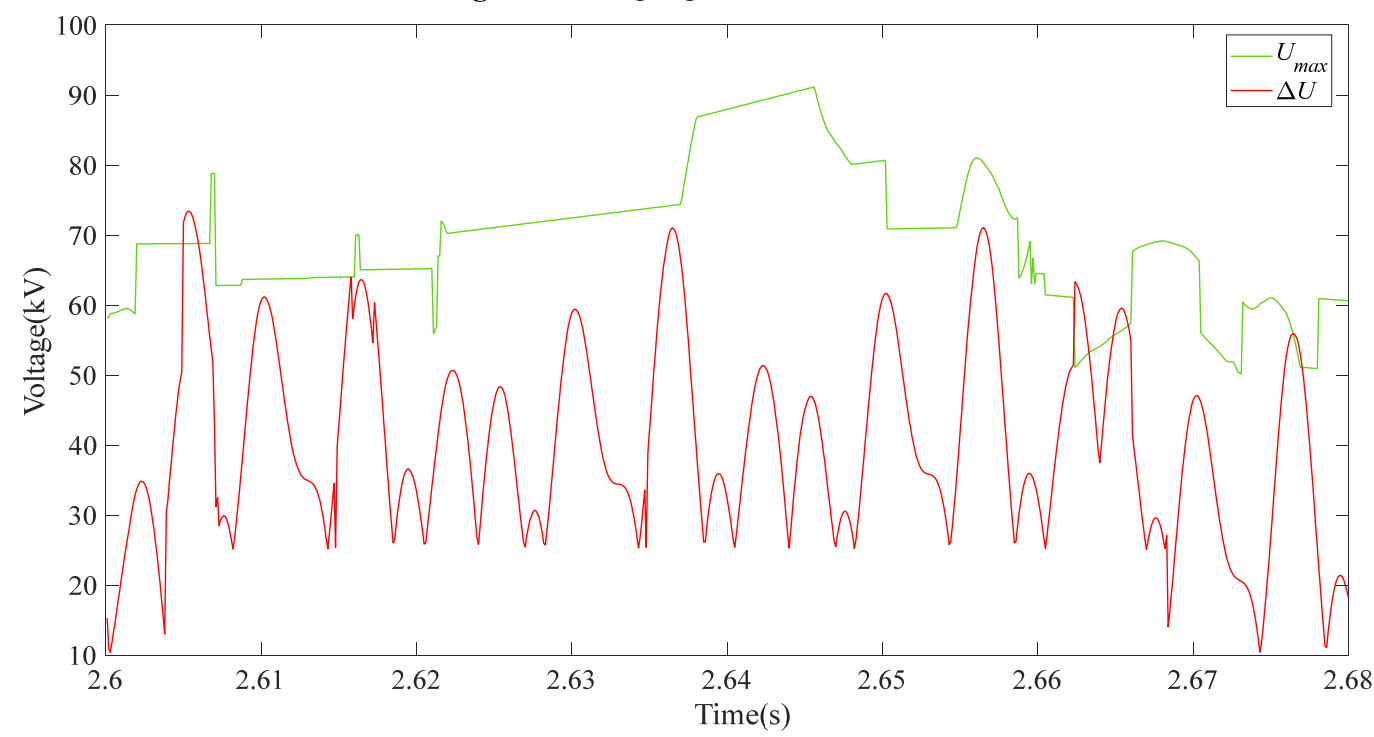

Figure 7. Schematic diagram of Simulation Results

\section{Conclusions}

Combining the commutation area theory and optimization method, this paper proposes a commutation failure evaluation method suitable for voltage waveform distortion conditions. Through theoretical analysis and simulation verification, the following conclusions can be drawn:

1) The influence of harmonics on the commutation process is a complicated process. The voltage waveform distortion caused by harmonics affects the size of the commutation area and the offset of the line voltage zerocrossing point. Therefore, the study needs to consider the comprehensive influence of amplitude and phase.

2) The evaluation method in this paper has the following advantages: (1) The AC voltage, which is one of the system state variables, has the characteristics of being easy to grasp and control in real time, and is suitable as a basis for evaluation. (2) The start-up criterion does not rely on simulation, has clear physical meaning, and ensures the reliability of prediction. (3) Because there are few data in the harmonic estimation data window at the beginning of the complex electromagnetic transient process, it is impossible to accurately calculate the harmonic parameters of each order. The algorithm uses the method of numerical integration and optimization to use the line voltage change as the judgment basis in order to avoid the error in the calculation of each harmonic.

3) This evaluation method does not consider the influence of the DC control system. The inverter side of the DC system contains a constant extinction angle control. When the extinction angle is too small, the control method is put into operation. It can suppress commutation failure to a certain extent by adjusting the firing angle. So the evaluation method has a certain degree of conservativeness.

\section{Acknowledgments}

I would like to give my sincere gratitude to Sucan, my colleague who, with extraordinary patience and consistent encouragement, gave me great help by providing me with necessary materials, advice of great value and inspiration 
of new ideas. Besides, I pleased to acknowledge Electric Power Research Institute of State Grid Hebei Electric Power Company for financial support to me, it is of great help for me to finish this research successfully.

\section{References}

1. Ling Xingyuan, Zhao Rui, Li Tianqi, et al. Research of Conventional High Voltage Direct Current Transmission System Stability Analysis and Control[J]. 2013,28(10):288-300.

2. Liu Jihao, Guo Chunyi, Liu Wenjing, et al. Commutation failure detective method based on improved commutation area in HVDC[J]. Journal of North of China Electric Power University, 2014, 41(1):15-21.

3. Zeng Liang, Li Yongli, Zhang Yunke, et al. Research on Mechanism and Control Strategy of Continuous Commutation Failures in HVDC System Caused by Asymmetrical Fault in Inverter-Side AC System, Proceedings of the CSEE,2019,11(1):3159-3168

4. Zhang L, Dofnas L.A novel method to mitigate commutation failures in HVDC systems[C]. International Conference on Power System Technology. IEEE, 2002.

5. Chen Shuyong, Li Xinnian, Yu Jun.et al. A method based on the sin-cos components detection mitigates commutation failure in HVDC $[\mathrm{J}]$. Proceedings of the CSEE, 2005,25,(14):1-6.

6. Guo C, Liu Y, Zhao C, et al. Power Component Fault Detection Method and Improved Current Order Limiter Control for Commutation Failure Mitigation in HVDC[J]. IEEE Transactions on Power Delivery, 2015, 30(3): 1585-1593.

7. Chang Peng, Research on Influence of Harmonics on the Failure of UHVDC Transmission[D]. Xinjiang University, 2017

8. Wang Haijun, Huang Yilong, Zhou Quan, et al. Analysis of commutation failure response strategies and prediction control technology in HVDC $[\mathrm{J}]$, Power System Protection and Control. 2014,42(21),124-131.

9. Rao Yufei, Zhang Penghui, Jiao Zaibin, et al. Mechanism and evaluating method for HVDC commutation failure caused by inrush current[J]. Power System Protection and Control, 2019, 47(13): 54-61.

10. Wang Feng, Liu Tianqi, Zhou Shengjun, et al. Mechanism and Quantitative Analysis Method for HVDC Commutation Failure Resulting From Harmonics. Proceedings of the CSEE, 2015, 35(19): 4888-4894.

11. Li Hui, The Analysis of Commutation Failure Prediction in UHVDC System[D]. Shanghai Jiao Tong University,2015. 